\title{
Von der Wissensgesellschaft zur empirischen Wissenschaftstheorie
}

\begin{abstract}
This paper argues that, from the perspective of the sociology of knowledge, science is not as clearly demarcated from other knowledge formations as a large part of science studies or the philosophy of science claim. Rather, the rise of the knowledge society not only results in a dissemination of science, scientific knowledge and practices into society, but also and consequently in a transgression of the boundaries of science itself. Since the boundaries of science become a problem, we need a new empirical theory of science, which combines the normative perspective of the Philosophy of Science with the descriptive, empirical and sociological perspective of Science Studies in order to critically clarify what we want to consider as scientific.

Zusammenfassung: Der Beitrag formuliert die These, dass die Wissenschaft aus der Perspektive der Wissenssoziologie keineswegs eine so klar abgegrenzte Wissensformation darstellt, wie die Wissenschaftstheorie, die Wissenschaftsforschung und auch ein Großteil der Wissenschaftssoziologie annehmen. Vielmehr ist sie mit dem Ausbau der Wissensgesellschaft selbst in die Gesellschaft diffundiert, sodass ihr Erfolg auch zugleich ihr Problem ist: Die Wissensgesellschaft zeichnet sich durch Verwissenschaftlichung und durch die Entgrenzung der Wissenschaft aus. Weil dadurch die Grenzen der Wissenschaft problematisch werden, ist eine neue empirische Wissenschaftstheorie erforderlich, die auf der Grundlage empirischer Beschreibung der Methoden wissenschaftlichen Forschens kritisch klärt, wie Wissenschaft sein soll.
\end{abstract}

\section{Einleitung}

Wissen im Widerstreit ist ein Thema, das die Wissenssoziologie besonders herausfordert. Sie behandelt die Verschiedenheit der Wissensbestände, Paradigmen, Ideologien - kurz: die Sozialität des Wissens, sodass für sie der Widerstreit des Wissens auch immer ein sozialer Konflikt ist. Anschaulich dargestellt wird ein solcher Streit derzeit in den Debatten um ,Fake News' und das Auftreten neuer Diskurse, in denen die bisher vorherrschende (Foucault'sche oder Habermas'sche) Kritik der Macht des Wissens zur Affirmation der Macht gegen besseres Wissen wird. Die neuen Diskurse gestalten sich derart, dass sie vormals machtkritische Perspektiven überspitzen und damit der Kritik am herrschenden Wissen einen Relativismus vorwerfen, den sie durch populistische Vereinfachungen ersetzen wollen: In unserem Fall um eine besondere Form des Wissens, nämlich die Wissenschaft. Wenn wir uns mit Wissenschaft beschäftigen, müssen wir einräumen, dass sie zwar systematisch einen Gegenstand der Wissenssoziologie bildet, sich die Wissenschaftsforschung in den letzten Jahrzehnten aber stark von der Wissenssoziologie 
abgekoppelt hat - mit zum Teil irritierenden Folgen, was die Verallgemeinerungen ihrer theoretischen Debatten angeht. Wenn ich hier also von der Wissenschaft, der Wissenschaftsforschung und der Wissenschaftstheorie rede, muss ich eingestehen, dass ich sie - gleichsam von außen - aus dem Blick der Wissenssoziologie betrachte. So sehr diesem Blick die ,Insider-Perspektive“ der (in Deutschland ohnehin nicht sehr stark verbreiteten) Wissenschaftsforschung fehlt (was die Thesenhaftigkeit dieses Beitrags erklärt, für die ich um Nachsicht bitte), öffnet er doch eine weitere Sicht auf die Lage der Wissenschaft, die gerade eine kaum auf die nationalen Grenzen beschränkte Gesamtgesellschaft darstellt. In der Tat möchte ich die These formulieren, dass die Wissenschaft keineswegs so abgegrenzt ist, wie die Wissenschaftstheorie, die Wissenschaftsforschung und auch ein Großteil der Wissenschaftssoziologie annehmen. Vielmehr ist sie mit dem Ausbau der Wissensgesellschaft selbst in die Gesellschaft diffundiert, sodass ihr Erfolg zugleich ihr Problem ist: Die Wissensgesellschaft zeichnet sich durch Verwissenschaftlichung aus; doch bedeutet die Diffusion der Wissenschaftlichkeit zugleich ein Problem für die Abgrenzung der Wissenschaft und zwar nicht nur institutionell, sondern auch hinsichtlich des institutionalisierten Handelns und Denkens: Was (gute) Wissenschaft ist, folgt - so kann man vermuten und befürchten - zunehmend externen Regeln und Prinzipien, die die Wissenschaft selbst dort nicht bestimmt, wo sie ihren immanenten Regeln zu folgen scheint. Deswegen, so meine Forderung, ist eine neue Wissenschaftstheorie nötig. Diese Wissenschaftstheorie kann nicht bei den vermeintlich einzeln erkennenden Forschenden beginnen, sondern muss ihren Ausgang bei der Wissenschaft nehmen, die offenkundig in Praxis und Diskurs ein soziales Unternehmen ist. Sie muss deswegen die empirische Erforschung der Wissenschaft ernst nehmen und - vor allem da, wo sie darüber reflektiert - die daraus gewonnenen Erkenntnisse zur Norm der eigenen Praxis machen. Dies ist es, was ich als empirische Wissenschaftstheorie bezeichne, da diese auf der Grundlage empirischer Beschreibung der Methoden wissenschaftlichen Forschens (und auf der Grundlage des normativen Charakters der Methoden) kritisch klärt, wie Wissenschaft sein soll.

Nachfolgend werde ich Sie zunächst ein wenig mit der Perspektive der Soziologie auf das Wissen vertraut machen (2). Auf dieser Grundlage werde ich dann die Wissensgesellschaft thematisieren (3), die auch diese Buchpublikation ausdrücklich anspricht. Ich glaube, dass dieser Begriff heute etwas irreführend ist, aber immerhin erlaubt er den Bezug auf die Rolle der Wissenschaft. Deren Abgrenzungsproblem wirft eine Frage auf, die als wissenschaftstheoretisch bezeichnet werden kann: Was ist Wissenschaft? Bei der Behandlung dieser Frage werde ich die These vertreten, dass die Ausweitung der Wissenschaft in die Wissensgesellschaft das Problem der Abgrenzung der Wissenschaft und des wissenschaftlichen Wissens verstärkt (4). Diese Frage, so werde ich argumentieren, fordert eine neue Wissenschaftstheorie, die noch nicht existiert, aber dringend erforderlich ist (5). 


\section{Wissen}

Warum, muss zuerst gefragt werden, nimmt sich denn die Soziologie des Wissens an, das von alters her eines der zentralen Themen der Philosophie ist? Dazu mag ein kurzer Rückblick hilfreich sein: Es ist nicht erst die weltweit berühmt gewordene deutsche Wissenssoziologie um Max Scheler und Karl Mannheim, die das Wissen in den Mittelpunkt stellt. Denn die Bedeutung des Wissens wurde schon vom Begründer der Soziologie, Auguste Comte, hervorgehoben. ${ }^{1} \mathrm{Zu}$ Beginn des 19. Jahrhunderts sah er die Besonderheit der anbrechenden modernen Gesellschaft nicht nur in der wachsenden Bedeutung der Industrie, sondern vor allem in der Umstellung vom religiösen und metaphysischen auf das positive (objektive) Wissen der Wissenschaft, dessen Krönung in seinen Augen die Soziologie darstellen sollte.

Auch Karl Marx erkannte die gesellschaftliche Bedeutung des Wissens an. Wissen ist seiner Vorstellung nach das Ergebnis eines aktiven Prozesses, der sich in der sozialen Praxis vollzieht. Die Ablösung der Erkenntnis von der Praxis in der modernen bürgerlichen Gesellschaft - etwa die ,Abgehobenheit‘ der universitären Wissenschaft von den Handlungsproblemen der Gesellschaftsmitglieder - ist für ihn eine Folge der Teilung von intellektueller und manueller Arbeit; sie bildet einen Grund für die „Entfremdung“, die sich durch die kapitalistische Produktionsweise noch verschärfen kann. In Verbindung mit den verschiedenen Klasseninteressen verweist diese Arbeitsteilung auch auf einen entscheidenden Aspekt seines Wissensbegriffes: In arbeitsteiligen Gesellschaften führt die materielle Ausbeutung dazu, dass das anerkannte Wissen zu einer Ideologie wird. Ideologie heißt jenes Wissen, das sehr entschieden vom Klassenstandpunkt derjenigen geprägt ist, die dieses Wissen vertreten und ihm durch ihre Herrschaft Legitimität verleihen; und dieses falsche Wissen prägt gar notwendig das Bewusstsein der Aktanten, wie er systematisch darlegt. In den ideologischen Wirren der 1920er Jahre bildete dieser Begriff die Grundlage für Karl Mannheims berühmte Fassung der Wissenssoziologie. Er hob die allgemeine „Standortgebundenheit“ des Wissens hervor: Nicht nur soziale Klassen, sondern jede Art sozialer Gruppierungen kultiviere ihr eigenes Wissen bzw. ihren „Denkstil“ und nehme dadurch eine besondere Perspektivität ein. Die Schöpfung der Wissenssoziologie ist in seinen Augen eine Reaktion auf dieses Problem der pluralen Perspektiven: Indem sie sich auf die Abhängigkeit des Wissens von den sozialen Standorten konzentriert, verfolgt sie das Ziel, deren Geltung ins (soziale) Verhältnis zu setzen oder, wie er es nannte, $\mathrm{zu}$,relationieren“.

Es wird noch eine Rolle spielen, dass sich diese wissenssoziologische Perspektive im Gefolge von Mannheim nicht nur in feministischen und postkolonialistischen Debatten wiederfindet, sondern auch auf die Wissenschaft ausgeweitet wurde. Hatte Ludwik Fleck schon zu Anfang der 1930er Jahre die Abhängigkeit biologischer

1 Ausführliche Hinweise und detaillierte Erläuterungen zu den folgend genannten Autoren und den Themen der Wissenssoziologie und Wissensforschung finden sich in Knoblauch 2014. 
„Denkstile“ von den Denkkollektiven am Beispiel der Syphilis-Forschung aufgezeigt, verband Anfang der 1960er Jahre Thomas Kuhn (1967) beide Begriffe zum berühmten Terminus des „Paradigmas“ vor allem an Beispielen aus den Naturwissenschaften: die Wissenschaft „akkumuliere“ keineswegs ständig ihr Wissen, wie etwa Popper (1935) angenommen hatte, sondern sei ständigen Veränderungen, ja Revolutionen, ausgesetzt, in denen altes Wissen völlig entwertet und ersetzt werde, da dessen Trägerschaften sich änderten. Spätestens mit dem „Strong Programme“ der Wissenschaftssoziologie begann sich innerhalb der zunehmend sozialwissenschaftlich orientierten Wissenschaftsforschung (,Science Studies') die Einsicht durchzusetzen, dass gerade die wissenschaftliche Erkenntnis entscheidend von ihrem sozialen Kontext beeinflusst werde. Im sogenannten „Laborkonstruktivismus“ wird gar die These vertreten, dass die Handlungen, mit denen die Wirklichkeit erforscht wird, zur Konstruktion eben dieser Wirklichkeit beitragen. ${ }^{2}$

Der Gedanke, dass jedes Wissen eine soziale Konstruktion sei, war zuvor in einer umwälzenden jüngeren wissenssoziologischen Theorie formuliert worden: In ihrer „gesellschaftlichen Konstruktion der Wirklichkeit“ hatten Peter Berger und Thomas Luckmann 1966 nicht nur eine der ersten Formulierungen des wissenschaftlichen Konstruktivismus geleistet, sondern als Theorie der Wissenssoziologie war ihr Buch auch ein radikaler Neubeginn der Wissenssoziologie. Neben dem wissenschaftlichen Wissen stehen auch politische Ideologien und religiöse Lehren, letztendlich alle Formen des Wissens, auch die des sogenannten Alltagsmenschen, in Wechselwirkungen zu den sozialen Einheiten, mit denen sie korrelieren und variieren. Noch grundlegender steht das weitgehend sozial vermittelte Wissen im Kern jeden Handelns, das es eben dieses Handeln als gesellschaftlich vermittelter Sinn leitet und durch die Folgen dieses Handelns selbst wieder zur Wirklichkeit wird. Obwohl das Wissen auf der Möglichkeit des Subjekts zum sinnhaften Handeln aufbaut, weist es einen entschieden sozialen Charakter auf: Erst im koordinierten Zusammenspiel mit anderen, dank der Vermittlung durch sie und kraft der mit ihnen gestalteten Institutionen, wird es zu ,unserem', das heißt kollektivem, sowie zu individuellem Wissen. Wissen weist eine unterschiedliche soziale Verteilung auf, die nach Klassen und Schichten variiert und entsprechend auch sozial unterschiedlich vermittelt wird. Es ist die Grundlage für die verschiedenen Institutionen, die sich etwa auf politisches, ökonomisches oder religiöses Wissen spezialisieren, und wird von diesen auch machtvoll umgesetzt; überdies beinhaltet es unterschiedliche Formen, die von den unausgesprochenen körperlichen Fertigkeiten über die expliziten Wissensbestände bis zu den ausformulierten symbolischen Legitimationen reichen, die auch den Nachgeborenen erklären sollen, warum es beispielsweise die Ehe, die Religion oder Deutschland gibt und geben sollte. Aus den Unterschieden der Macht verschiedener Institutionen und ihres Wissens sowie der sozialen Lage der Wissensträger lassen sich auch die Dynamiken und Konflikte, etwa zwischen konkurrierenden Eliten mit ihren je eigenen Legitimationen oder zwi-

2 Beispielhaft dafür Knorr-Cetina 1984. 
schen Experten und Alltagswissen - also zwischen den sozialen Wissensbeständen bzw. Wissensformationen -, erklären, von denen in diesem Band die Rede ist.

\section{Wissensgesellschaft und Wissenschaft}

Ich kann hier nicht in die Feinheiten der Wissenssoziologie eingehen, doch sollte aus dem bereits Gelesenen deutlich hervorgehen, dass aus ihrer Perspektive eigentlich jede Gesellschaft eine Wissensgesellschaft ist. Die jüngere Rede von der modernen, gegenwärtigen Wissensgesellschaft ist jedoch nicht tautologisch, sondern hat sehr stark mit der Änderung der gesamtgesellschaftlichen Produktion und der damit verbundenen Produzentenklassen in den letzten Jahrzehnten zu tun. $\mathrm{Zu}$ nennen ist der zunächst in westlichen Gesellschaften beobachtete Wandel von einer industriell dominierten Warenproduktion zur „Wissensarbeit“, der sich auf der ökonomischen Seite als Deindustrialisierung und sozialstrukturell durch das rasante Wachstum der „Wissensklasse“ auszeichnet. ${ }^{3}$ (In vielen nichtwestlichen Gesellschaften hat sich eine (ggf. als eigene Sektion separierte) Wissensgesellschaft entwickelt, ohne dass vorher eine Industrialisierung vorlag, was deutliche Differenzen der Mentalitäten bedingt.) Die Entlassung der Arbeiterschaft aus den handarbeitenden mechanischen, elektrischen und chemischen Industrien ist auch mit der Diagnose der „Dienstleistungsgesellschaft“ belegt worden, die durchaus ihre Berechtigung hat. Dass wir andererseits zusätzlich von Wissensgesellschaft reden, hat damit zu tun, dass sich die Rolle der Wissensvermittlung massiv geändert hat. Nehmen wir als Beispiel etwa die sogenannte „Bildungsrevolution“, die seit dem Zweiten Weltkrieg zuerst in den USA, nunmehr auch in Westeuropa, dazu geführt hat, dass ein immer größerer Teil der Menschen 10, 15 oder 20 Jahre in den Bildungssystemen verbringen, wenn sie nicht gar durch lebenslanges Lernen in Seminaren, Kursen oder auch digital lernen, ohne dass hier noch von Weiterbildung gesprochen werden müsste. Mit dieser massiven Zunahme an Bildung haben sich auch die formalen Kriterien des Bildungssystems zunehmend durchgesetzt: Arbeit in einem qualifizierten Sinn ist selbst im handwerklichen Bereich nicht mehr ohne das möglich, was eigentlich als akademischer Abschluss gelten müsste: von Berufsschulen über Berufsakademien bis hin zu den Fachhochschulen und Universitäten, deren BA und andere Abschlüsse mittlerweile sogar ein transnational gültiges, symbolisches Kapital' (im Sinne Bourdieus) darstellen. Wegen dieser formalen Auszeichnungen ist schon seit den 1960er Jahren von einer Wissensklasse gesprochen worden. Der Begriff hat sich zwar nicht durchgesetzt, doch folgt man den Analysen des amerikanischen Soziologen Fligstein (2008), könnten wir die gegenwärtige Attacken des europäischen Rechtspopulismus durchaus als eine Reaktion auf die Ausbildung einer europäischen, mehrsprachigen, internationalistischen sozusagen „Erasmus-Wissensklasse“ ansehen.

3 Vgl. zur Diskussion um die Wissensgesellschaft Engelhardt/Kajetzke 2010. 
Die Wissensgesellschaft zeichnet sich zudem dadurch aus, dass sich immer mehr Leute um das Wissen anderer kümmern: Wissen als Wissen wird zum Gegenstand menschlicher Kommunikation, menschlicher Arbeit und damit auch zur Ware. Die Vorstellung der Wissensgesellschaft zehrt aber nicht nur von der Wissensvermittlung im Bildungssystem, sondern es spielt vielmehr die Produktion von Wissen als Wissen eine zentrale Rolle und damit auch die Wissenschaft. Wir können deswegen von einer Verwissenschaftlichung sprechen. Diese Verwissenschaftlichung ist erkennbar an der zunehmenden Durchdringung auch der nichtwissenschaftlichen gesellschaftlichen Funktionsbereiche (Wirtschaft, Politik, Religion, Medien, Sport etc.) mit wissenschaftlichem Wissen. Einige Autoren sehen in dieser Verwissenschaftlichung die Ursachen für deren allmähliche Transformation, etwa zur Wissensökonomie, zur Wissenspolitik etc. (z. B. Stehr 2002).

So sehr das Wissen ein Schlüssel für Berufe ist und damit den Zugang in den Kern anderer sozialer Systeme - wie zu den Positionen in der Wirtschaft oder in der Religion - öffnet, so unangemessen wäre es, die Wissenschaft als eine Art Leitsystem der Wissensgesellschaft anzusehen. Zweifellos ist, dass die Wissenschaft das symbolische Kapital des gesellschaftlichen Wissens legitimiert. Das zeigt sich relativ einfach und grob daran, dass der Wert des Wissens mit seiner Wissenschaftlichkeit steigt. Wenn wir nur die Abschlüsse etwa zwischen Arzthelfern und Ärztinnen oder Hauptschülerinnen und Promovierten ansehen, dann lässt sich leicht erkennen: Je näher das erworbene Wissen der Wissenschaft kommt, umso mehr scheint es wert zu sein - wenngleich nicht mehr erwartbar von jedem ,wertvollen' Wissen Gebrauch gemacht wird und das intellektuelle Prekariat anzuwachsen droht. Allerdings darf der Einfluss anderer Institutionen auf die Wissenschaft nicht übersehen werden. Abgesehen davon, dass etwa die Politik zumindest in Deutschland weniger als etwa in Frankreich von formalen Wissensabschlüssen abhängt, kann die Wissenschaft weder ökonomisch noch politisch als eine leitende Institution angesehen werden. Vielmehr finden wir eine Reihe von massiven Einflüssen anderer Systeme auf die Wissenschaft.

Wenn wir etwa das Verhältnis der Wirtschaft zur Wissenschaft betrachten, dann geht es nicht nur um den riesigen Bereich von „Forschung und Entwicklung“, neuerdings auch als „Innovation“ bezeichnet, sondern vielmehr darum, dass die ökonomische Logik bekanntlich auf die Wissenschaft zugreift, wie durch die Einführung des New Public Management, Wettbewerbs- und Belohnungsverfahren deutlich geworden ist. Aber auch die Politik hat über die Vergabe von Forschungsmitteln, die Besetzung von Stellen und das damit ermöglichte ,Agenda-Setting“ wissenschaftlicher Forschungsthemen (von der Nachhaltigkeit über die ,digitale Gesellschaft ‘ bis zur ,Künstlichen Intelligenz') einen Einfluss - von den Massenmedien und den neuen Medien ganz zu schweigen, die ja mittlerweile schon so sehr in die Wissenschaft eingebaut sind, dass klare Grenzziehungen kaum mehr möglich scheinen.

Die Verwissenschaftlichung der Gesellschaft ist also janusköpfig, denn sie geht notwendiger- und logischerweise mit einer Entgrenzung der Wissenschaft einher, die im Begriff der Wissensgesellschaft ihren Ausdruck findet. Ohne hier auf die einzelnen organisationalen Zusammenhänge eingehen zu können, ist unübersehbar, dass 
Politik (Staat, Parteien, Stiftungen), Wirtschaft (private Forschungseinrichtungen, Finanzierungen), Kirchen (Theologische Fakultäten, Forschungsinstitute), Medien (Wissenschaftsjournalismus) und viele andere Institutionen auf die Wissenschaft zugreifen und sie bis in die Forschungsthemen (und der Forderung nach Nützlichkeit) hinein bestimmen. Wir haben es also mit einer Reihe von Tendenzen zu tun, die gerne übersehen werden, wenn die Wissenschaft wie ein geschlossenes System betrachtet wird: Die zumeist von außen betriebene Förderung der Interdisziplinarität wird durch für andere Systeme nützliche Transdisziplinarisierung so ausgeweitet, dass die Grenzen zwischen Wissenschaft und Politik ebenso unscharf werden, wie die zwischen wissenschaftlicher und zum Beispiel tänzerischer oder anderer künstlerischer Forschung, was mit einer Verflüssigung ihrer Strukturen etwa durch Projektifizierung einhergeht (vgl. Baur/Besio/Norkus 2016). Aus der Beobachtung solcher Entwicklungen entspringt dann etwa die postmoderne Diagnose einer neuen Wissenschaft, die als „Mode 2 “ bezeichnet wird (Gibbons et al. 1994).

In der Tat macht sich eine Unzahl an legitimatorischen Formaten breit, die die Wissenschaft nach außen vermitteln und die von ,Public Understanding of Science‘ (PUS) über ,Public Science‘ bis zu ,Lay Science‘ reichen. Durch die Ausbreitung von neoliberalen Methoden des ,Public Management ‘ beobachten wir einen Einzug der verschiedensten Formate und Formen aus der Ökonomie in die Wissenschaft: Die Evaluation über ökonomische Kriterien und Einkünfte, die Konsumbewertung von Lehrleistungen und, nicht zuletzt, die urökonomischen Präsentationsformen etwa von PowerPoint oder Science Slams, die mittlerweile im Herzen der wissenschaftlichen Kommunikation zu finden sind (vgl. dazu Knoblauch 2013; Wilke/Hill 2019). Die Ausbreitung neuer Finanzierungsmodelle für wissenschaftliche Veröffentlichungen oder die massive Ausweitung wissenschaftlicher Datenbanken (etwa im Zuge des Ausbaus nationaler Forschungsdateninfrastrukturen) und die Integration von Forschungsmanagementsystemen in die Projektarbeit sind Beispiele dafür, dass die Digitalisierung weitere Veränderungen bewirken wird, die soweit ich sehen kann - ihre Motivation nicht aus der Wissenschaft ziehen, sondern eher aus anderen Bereichen übernehmen (vor allem aus dem ökonomischen Bereich).

Bedenken wir, dass etwa die Universitäten, die ja zentrale Einrichtungen der Wissenschaft darstellen, hierzulande gleichzeitig als Ausbildungsort für mehr als ein Drittel des Jahrgangs der ganzen Bevölkerung dienen, die weder motivational noch (bei den gegebenen Ressourcen) strukturell (durch „Verbindung von Forschung und Lehre“) ernsthaft zu „Wissenschaftlerinnen und Wissenschaftlern“ herangebildet werden können, so ist die oben angedeutete Ausweitung und zuweilen auch Inflationierung des Begriffes „Forschung“ verständlich und es erscheint als sehr fraglich, ob Wissenschaft wirklich sinnvoll a priori als ein abgeschlossenes Subsystem angesehen werden kann. Mir geht es hier keineswegs um ein Plädoyer für eine postmoderne Entgrenzung der Wissenschaft, sondern vielmehr um die Rettung der Wissenschaft vor dieser Entgrenzung. Denn die Wissenschaft läuft meines Erachtens zunehmend Gefahr, zum Teil einer breiteren Wissensformation zu werden, in der durchaus sehr andere Orientierungen oder „Codes“ als die der Wahrheit am Werke sind. Dabei treten 
Überschneidungen zwischen den „Funktionen“ und vor allem auch dominierenden Machtinteressen nicht nur der verschiedenen Institutionsbereiche auf (etwa zur staatlich finanzieren Auftragsforschung, zu Erkenntnisinteressen von Großkonzernen oder auch von Kirchen und Sportverbänden); aus der Perspektive der Wissenschaft mit ihrem historisch entwickelten Selbstverständnis als anerkannte und symbolisch hoch bewertete Institution entstehen zudem auch Spannungen und Konflikte in diesen Übergangsbereichen, die durchaus der - von Bourdieu (2001) entwickelten - Logik des sozialen Feldes folgen, wie es etwa von Münch (2007) für Deutschland rekonstruiert wird. Stellen wir es vereinfacht dar, ohne die Einflüsse anderer Systeme $\mathrm{zu}$ berücksichtigen, dann finden wir am einen Pol eine mehr oder weniger dominierende „Grundlagenwissenschaft“, von dort aus aber zunehmend diverse Öffnungen in und Interdependenzen mit anderen Institutionen der Gesellschaft (siehe Abb. 1).

\section{Wissenschaft: Institutionelle Spezialisierung, Differenzierung, Fragmentierung,} ,Fraktalisierung، (ausgewählte Beispiele)

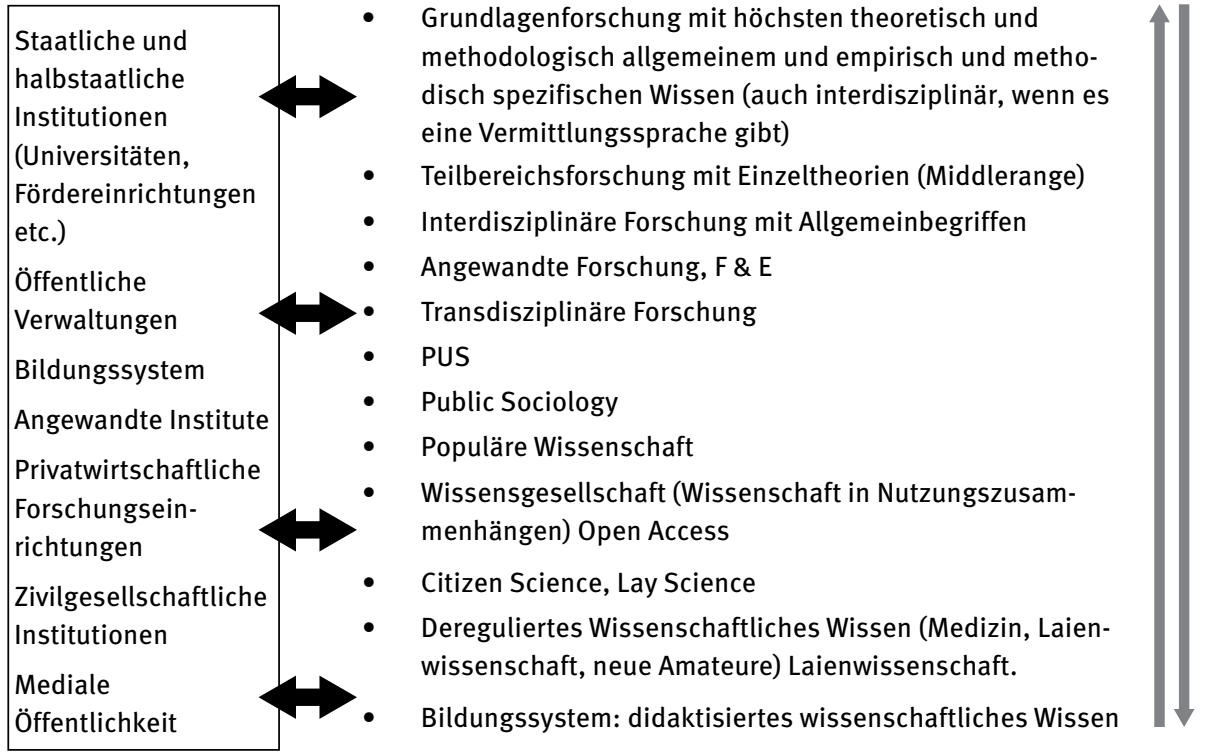

\section{„Verwissenschaftlichung“ des Altagswissens/ Mediatisierung des Wissens Wissensgesellschaft}

Abb. 1: Das „System der Wissenschaft“ und dessen Wechselwirkungen mit anderen Institutionen der Gesellschaft

Zusätzlich, wie allerdings Bourdieu selbst schon Entwicklungen zur Entgrenzung sozialer Felder angedeutet hat, scheint das Feld der Wissenschaft ganz entscheidend durch seine (durchaus selektiv angelegte) Internationalität entgrenzt, die etwa in den internationalen Rankings und dem damit keineswegs direkt verbundenen international-nationalen Reputationsgefälle erkennbar ist. Dessen ungeachtet haben wir es 
jedoch eben auch mit einer zunehmenden Durchmischung wissenschaftlicher Kerninstitutionen mit anderen gesellschaftlichen Aufgaben und vor allem Umwertungen zu tun, die die Frage nach den Grenzen des Feldes aufwerfen - und damit die Existenzfrage danach, was die Wissenschaft eigentlich ist.

\section{Die Grenzen der Wissenschaft und die empirische Wissenschaftstheorie}

Die wissensgesellschaftliche Ausweitung weist auf ein Problem hin, das seit Popper im Kern der Wissenschaftstheorie steht: Wie lässt sich Wissenschaft abgrenzen? Diese Frage bezieht sich nicht nur auf die Wissenschaft als Institution, wie wir eben skizziert haben, sondern auch auf die institutionalisierten Handlungen und damit auf das Wissen. Sie wirft also die Frage auf: Was zeichnet wissenschaftliches Wissen (noch) aus?

Diese Frage scheint mir derzeit vor allem auf eine pragmatische Weise gelöst $\mathrm{zu}$ werden: Die pragmatische Lösung kümmert sich nicht um die Frage, wie Wissenschaft abgegrenzt werden kann, sondern konzentriert sich auf das, was funktioniert und nützt. Das geschieht zwar sehr häufig und, vor allem in der Kombination mit der Technik, sehr effizient, wird aber der Wissenschaft vermutlich im Kern nicht gerecht. Wenn die Wissenschaft darin besteht, dass sie angeben können muss, wie sie ihr Wissen erzeugt hat, und dass andere dies nachvollziehen können, dann ist diese Antwort nicht nur ignorant, sondern auch naiv, denn sie gibt dieses Prinzip der Nachvollziehbarkeit auf, sondern wird unter den gegebenen Bedingungen der Wissensgesellschaft auch zum Spielball aller anderen Institutionen und ihrer Interessen: Was ,richtig', ,nützlich' oder relevant ist, entscheidet dann die Politik, die Wirtschaft, möglicherweise der Präsident der Vereinigten Staaten.

Daneben gibt es, wie wir sehen, auch eine ,fundamentalistische‘ Lösung auf diese Frage. Fundamentalistisch ist ein legitimatorischer Bezug zur klassischen Wissenschaft: Wir kehren zu den alten, bewährten Modellen, Methoden und Theorien der klassisch-modernen Wissenschaft zurück und ignorieren die gegenwärtig so fragmentierte und ,fraktale‘ Forschung und Diskussion. Dieses Modell kann allerdings nur durchgesetzt werden, wo Tradition oder Autorität eher rituell als kritisch bestimmen, was Wissenschaft ist, ohne die tatsächlichen Veränderungen ihrer Institutionen, ihrer Handlungsweisen und ihrer Gegenstände zu berücksichtigen.

Mein Vorschlag möchte sich für eine dritte Variante aussprechen: Die Wissenschaft reflektiert selbst auf das, was sie sein soll, indem sie beobachtet und mit ihren ureigenen Methoden der Kritik bestimmt, was sie als Wissenschaft auszeichnet. Dieser Vorschlag ist keineswegs ganz neu, verbindet er doch zwei Ansätze, die jedoch bisher noch nicht verbunden waren. Wie der Hinweis auf Popper zeigt, gibt es schon lange eine „Wissenschaftstheorie“, deren Aufgabe es im Grunde ist, zu bestimmen, 
was wissenschaftliches Wissen auszeichnet. Genauer genommen aber handelt es sich um eine Wissenschaftsphilosophie, und so heißt das Fach im englischen Sprachraum - wo der Begriff der Wissenschaftstheorie wenig bekannt ist - auch durchgängig „Philosophy of Science“. Die Wissenschaftsphilosophie hat sich etwa mit der Bedeutung von Induktion und Deduktion, der Rolle von Erklären oder der Differenz von Sozial- und Naturwissenschaften beschäftigt. Weil sie immer auch Methodologie, also Logik der Forschung, ist, hat sie eine normative Komponente (Mittelstraß 1974). Normativ bedeutet hier keine Einführung außerwissenschaftlicher, gesellschaftlicher Normen, die sich (zumeist und zu Recht) für eine gute, demokratische Gesellschaft auch in der Wissenschaft einsetzen. Bedenken wir, dass die schiere wissenschaftliche Forderung zur Einhaltung von wissenschaftlichen Methoden aus soziologischer Sicht selbst schon als normativ gelten kann, so geht es hier eben um die Frage nach der spezifischen Normativität, die in der besonderen Praxis, im spezifischen Wissen und im (diskursiven) Selbstverständnis des wissenschaftlichen Handelns selbst liegt. ${ }^{4}$

Wir sehen schon an den Beispielen, dass es der Wissenschaftsphilosophie vor allem um das richtige Denken geht, und genau hier liegt auch ihr Problem: Denn die Wissenschaftsphilosophie betrachtet die Wissenschaft im Wesentlichen nach dem Modell der klassischen Erkenntnistheorie. Das heißt, dass sie von individuellen Subjekten ausgeht, die eine Erkenntnis haben, die Schlüsse vollziehen und die auch diese Vollzüge dann individuell an andere Individuen kommunizieren. Diese Beschränkung wirkt sich auch auf die Wissenschaftsphilosophie selbst aus. Nach einer längeren Hochphase wird ihr Zustand heute als „gloomy“ (Agassi 2011: 280) beschrieben. Nach ihren Hochzeiten bis zu den 1980er Jahren (und dem Erscheinen etwa der „Enzyklopädie für Philosophie und Wissenschaftstheorie“) wird sie zwar glücklicherweise in der Philosophie noch betrieben, doch stellen selbst Insider fest, dass „exchanges between philosophy of science, history of science and science studies have been rather sparse; in fact, the disciplines have drifted further apart" (Schickore/Steine 2006: ix). Weingart (2003: 12) bemerkt, dass die Unterschiede zwischen der formalen Orientierung der philosophischen Wissenschaftstheorie und der sozialwissenschaftlichen Wissenschaftsforschung ebenso groß seien wie ihre institutionellen Distanzen. Ammon (2011: 1) schließt daraus, dass die Verbindung zwischen Wissenschaftsgeschichte, Wissenschaftsphilosophie und Wissenschaftssoziologie noch immer ein Desideratum sei - von der Wissenssoziologie ganz zu schweigen.

An die Stelle der Wissenschaftsphilosophie tritt immer häufiger eine andere Forschungsrichtung, die einen sehr engen Bezug zur anfänglich skizzierten Wissenssoziologie hat. Im Gefolge der schon einleitend beschriebenen Wissenschaftssoziologie hat sich vor allem im englischen Sprachraum ein Bereich ausgebildet, der als „Sociology of Scientific Knowledge“, „Social Studies of Science“ oder auch „Science

4 Beispielhaft dafür sind die ethischen Imperative, die Merton 1972 in der Wissenschaft vermutete, wie etwa Universalismus, Gemeinschaftlichkeit oder „Kommunismus“, Uneigennützigkeit und organisierter Skeptizismus. 
and Technology Studies“ bezeichnet wird. Dabei handelt es sich um historische und sozialwissenschaftliche empirisch ausgerichtete Arbeiten, die sich mit dem wissenschaftlichen Wissen als einem sozialen Phänomen beschäftigen. Diese soziologisch inspirierte empirische Wissenschaftsforschung hat (wie im zweiten Abschnitt mit Verweis auf Kuhns Paradigmen bereits angedeutet) gezeigt, wie problematisch die Annahme eines linearen Wissensfortschritts bzw. einer Akkumulation des Wissens ist, von der traditionalistisch an Popper anschließende Wissenschaftstheorien auch heute noch ausgehen. Ebenso sehr hat sie verdeutlicht, wie sehr die Wissenschaft aktiv an der Konstruktion der Tatsachen beteiligt ist, die erkenntnistheoretisch als unabhängig von ihr dargestellt werden. Schließlich aber hat sie ein anderes Modell der Erkenntnis bzw. des Wissens vorgeschlagen, das sich von dem der Wissenschaftstheorie grundlegend unterscheidet. Wissen wird hier als etwas betrachtet, das erst in einer Relation mit anderen eine Rolle spielen kann. Es ist ein soziales Wissen, das vor dem Hintergrund von Unausgesprochenen besteht und dank einer Form des Handelns erst in die soziale Welt tritt, die wir als kommunikativ bezeichnen müssen. ${ }^{5}$ Gerade wissenschaftliches Wissen besteht erst in der Objektivierung, die häufig Geräten zu verdanken sind, die helfen, sie in Zeichen und Diskurse zu transformieren. Zeichen und Diskurse erlauben die Kommunikation des Wissens und damit deren Faktizität; deren Geltung bedarf sozialer Netzwerke und Institutionen, in denen die Konventionen der Wissenschaft erlernt und tradiert werden. Dazu kommt noch eine Reihe von ökonomischen, politischen oder auch juristischer Rahmenbedingungen, die sie institutionell und bis in die Handlungen hinein mitprägen.

So materialreich und nachvollziehbar die empirische Wissenschaftsforschung die Relativität der wissenschaftlichen Forschung aufzeigen konnte, so wenig beschäftigt sie sich selbst allerdings wiederum mit der Frage, wie ihre eigenen Aussagen der Relativität entkommen. Dies liegt vermutlich auch daran, dass diese Wissenschaftsforschung bislang stark analytisch-beschreibend geblieben ist und dort, wo sie Normen und Werte anspricht, vor allem Bezüge herstellt, die nicht spezifisch für die Wissenschaft sind. So relevant es ist, die Rolle von Gender, sozialer Ungleichheit und Herrschaftsstrukturen zu berücksichtigen, so sehr rückt dabei die Frage nach dem Spezifischen der Wissenschaft in den Hintergrund. Die Wissenschaftsforschung hat gezeigt, was die Wissenschaft ist, nicht aber, wie sie als Wissenschaft sein soll.

Als Lösung auf diese paradoxe Situation erscheint es sinnvoll, die normative Orientierung der Wissenschaftstheorie aufzunehmen und sie mit der Wissenschaftsforschung so zu verbinden, dass sie die Normen des richtigen Forschens, der Orientierung am Gemeinsamen und die Befunde des Objektiven selbst zum Gegenstand macht. Dabei sollte man beachten, dass es hier nicht nur um die (durchaus beachtenswerte, aber etwa von Bourdieus „reflexiver Soziologie“ aufgenommene) Orientie-

5 Diese alte wissenssoziologische Einsicht wird in der jüngeren Philosophie als „,social epistemology“ bezeichnet (Schmitt 1994). Zum kommunikativen Handeln und der kommunikativen Konstruktion vgl. etwa Knoblauch 2017. 
rung an außerwissenschaftlichen gesellschaftlichen Normen geht, deren Bedeutung unzweifelhaft ist. Es geht hier auch um die Frage nach Normen und Werten, die der Wissenschaft, ihren Tätigkeiten und ihren Orientierungen zeitweilig, teilweise oder potentiell immanent sind und in ihren Handlungen und Institutionen wiederum umgesetzt werden sollen. Diese Frage wurde angegangen, doch kann sie begründet nur beantwortet werden, wenn sich die Wissenschaftsphilosophie mit der empirischen Wissenschaftsforschung verbindet und vice versa. Es geht also nicht um die Austreibung der Wissenschaftsphilosophie, sondern um das Projekt einer interdisziplinären Wissenschaftsforschung, die nicht nur anerkennt, dass die Wissenschaft ein durch und durch soziales und kulturelles Unternehmen ist, sondern dass die Wissensproduktion sowohl in den Entdeckungs- als auch in den Rechtfertigungskontexten sozial organisiert ist. Das ist eine empirische Wissenschaftstheorie, der es nicht nur um die „Beobachtung“ der Wissenschaft, sondern um die reflektierte (gute) Praxis geht.

Ich kann hier keine breite Skizze der empirischen Wissenschaftstheorie vornehmen, wie sie entwickelt werden soll, ${ }^{6}$ möchte aber wenigstens vier ihrer Grundsätze formulieren:

- Die empirische Wissenschaftstheorie muss empirisch sein: sie muss beobachten, wie die Wissenschaft wirklich betrieben wird; dabei sollte sie nicht übersehen, dass Wissen zwar sozial ist (also historisch „machtvoll“ institutionalisiert, von praktischen und legitimierenden Diskursen erfüllt und in gesellschaftliche Strukturen eingebettet), aber ohne Subjekte nicht verstehbar ist.

- Deswegen kann und muss sie reflexiv sein: sie muss also sich selbst beobachten und zwar auch und besonders hinsichtlich ihrer eigenen methodischen Vorgehensweisen, die ihre Wissenschaftlichkeit auszeichnen; ${ }^{7}$ so sehr die Wissenschaft davon lebt, dass sie die Welt beschreibt, so sehr liegt ihre privilegierte Position darin, dass sie angeben kann, wie ihre Beschreibung zustande kommt.

- Die empirische Wissenschaftstheorie muss kritisch sein. Im Unterschied zur Politik etwa, die Kritik ebenso übt, nimmt die Kritik in der Wissenschaft eine Sonderstellung ein, denn die Wissenschaft muss die Methoden der Herstellung ihrer Aussagen angeben, wie sie begründet und entsprechend kritisiert werden kann. Diese Methode der Kritik ist institutionell tief in die Wissenschaftsgeschichte eingeschrieben und daher ist es eine der wesentlichen Züge einer solchen Wissenschaftstheorie, dass sie die empirisch beschriebene Wissenschaft gegen ihre eignen Ideal abgleichen kann.

- Ausgehend vom sozialen Wissensbegriff kann Kritik aber nicht auf den subjektiven Nachvollzug beschränkt, sondern muss kommuniziert werden. Um zu vermeiden, dass dabei nur wieder eine Spezialdisziplin ausdifferenziert wird, die

6 Ein etwas weiter entwickelter Entwurf findet sich in Knoblauch (im Druck).

7 Die Vorgehensweise mit Blick auf qualitative Methoden der empirischen Sozialforschung ist skizziert in Knoblauch 2018. 
sich neben andere stellt und Wissenschaft weiter fragmentiert, scheint es nahezuliegen, eine besondere kommunikative Form zu entwickeln, die sich an die verschiedensten institutionellen wissenschaftlichen Zusammenhängen anpassen lässt. Als Beispiel einer solchen Form kann etwa Habermas’ „Seminarmodell der Kommunikation“ dienen, das eigentlich für jede gesellschaftliche Kommunikation entwickelt wurde, nun allerdings - auf der Grundlage empirisch bewährter Kommunikationsformen - auf die besonderen Anforderungen der Wissenschaft und ihrer Kritik zugeschnitten werden muss. Die genauere Gestaltung ist eine der Aufgaben der empirischen Wissenschaftstheorie.

\section{Fazit}

Dieses hier vorgeschlagene Konzept der empirischen Wissenschaftstheorie als eines kritischen Diskurses über das, was an der Wissenschaft empirisch beobachtbar ist, unterscheidet sich sowohl vom klassisch modernen ,Objektivismus ' wie auch von den postmodernen Theorien, die Wissenschaft als Palaver betrachten (Stengers 2008: 176), das lediglich situativ kontingente, bestenfalls plausible Deutungen produziert und seinen Wahrheitsbegriff partizipativ mit allen (aber auch wirklich allen) teilen muss. Eine Wissenschaftstheorie, die sich nicht nur auf die sprachlich kommunikativen Handlungen beschränkt, sondern sie im materialen und verkörperten Vollzug empirisch betrachtet, kann nicht übersehen, dass es sich dabei um eine (durchaus auch im globalen Raum westlich geprägte) sozial konstruierte objektivierte Wirklichkeit handelt, die auch die Formen der Kommunikation über die Wissenschaft prägt. Schon zu ihrer Reproduktion übernimmt sie zweifellos nicht nur wissenschaftliche, sondern auch wirtschaftliche, politische und öffentliche Aufgaben ebenso wie Bildungsaufgaben.

Deswegen erscheint es nur konsequent, dass es ein „public understanding of science“ bzw. eine „public sociology“, eine partizipative Forschung und eine populäre Wissenschaft geben muss, die an ein breites Publikum adressiert sind, und dass die Wissenschaft in einer demokratischen Gesellschaft öffentlich aktiv sein muss. Gerade aber weil die Wissenschaft diese vielfältigen Funktionen erfüllt, muss sie sich auch vor Übergriffigkeit schützen und - als Wissenschaft eben durch kritische Diskurse, die sich auf empirische Daten stützten - ihre Eigenheit bewahren. Denn greift schon die Wirtschaft mit ihren Stiftungsprofessuren, Projektgeldern und Qualifikationsanforderungen für Berufe auf Inhalte sowie Formen von Forschung und Studium $\mathrm{zu}$, tun dies ebenso die politischen Parteien - die der Wissenschaft wohlgesonnenen wie auch die sie beschneidenden, die über Ministerien, aber auch wissenschaftliche Ausschüsse die ,Nützlichkeit' der Wissenschaft erzwingen oder zumindest ihre innere Ordnung (etwa mit dem Druck auf interdisziplinäre Kooperationen) beeinflussen und bis tief in die wissenschaftliche Diskussionen und ihre Strukturen und Argumente hin- 
einwirken. Soll die Wissenschaft nicht einfach zum Spielball dieser Zugriffe werden, muss sie sich darüber verständigen, was sie als Wissenschaft ausmacht (falls sie nicht durch nicht begründete Formen der Herrschaft bestimmen lassen möchte, was sie zu tun hat). Auch wenn die Gefahr besteht, dass sich ein großer Teil der Bemühungen auf die Vermittlung zwischen den Disziplinen richten könnte, und auch wenn diese Verständigung derzeit in unzählige fraktale Diskurse zerfällt, müssen diese möglichst dauerhaft, breit und übergreifend geführt werden, wenn sich die Wissenschaft als Wissenschaft behaupten will.

\section{Literatur}

Agassi, J. (2011). Current Philosophy of Science. Philosophy of the Social Sciences 41(2), 278-294.

Ammon, S. (2011). Knowledge, the Context Distinction and its Impact on the Relation between Philosophy and the Sociology of Science. In: K. François et al. Hrsg. Foundations of the Formal Sciences VII: Bringing Together Philosophy and Sociology of Science (Studies in Logic 32). London: College Publications, 1-16.

Baur, N., C. Besio und M. Norkus (2016). Organisationale Innovation am Beispiel der Projektifizierung der Wissenschaft. In: W. Rammert et al. Hrsg. Innovationsgesellschaft heute. Wiesbaden: Springer, 373-402 [DOI: 10.1007/978-3-658-10874-8].

Berger, P. L. und T. Luckmann (1969). Die gesellschaftliche Konstruktion der Wirklichkeit: Eine Theorie der Wissenssoziologie. Frankfurt/Main: Fischer [1 $1^{\text {st }}$ English edition 1966].

Bourdieu, P. (2001). Science de la science et réflexivité. Paris: Raisons d’Agir.

Engelhardt, A. und L. Kajetzke. Hrsg. (2010). Handbuch Wissensgesellschaft: Theorien, Themen und Probleme. Bielefeld: transcript.

Fligstein, N. (2008). Euroclash: The EU, European Identity, and the Future of Europe. Oxford, New York: Oxford University Press.

Gibbons, M. et al. Hrsg. (1994). The New Production of Knowledge: The Dynamics of Science and Research in Contemporary Societies. London: Sage.

Habermas, J. (1981). Theorie des kommunikativen Handelns. Frankfurt/Main: Suhrkamp.

Knoblauch, H. (2013). Powerpoint and the Communication Society. Cambridge: Cambridge University Press.

Knoblauch, H. (2014). Wissenssoziologie. Konstanz: UVK, 3.Aufl. [1. Aufl. 2005].

Knoblauch, H. (2017). Die kommunikative Konstruktion der Wirklichkeit. Wiesbaden: Springer [DOI: 10.1007/978-3-658-15218-5].

Knoblauch, H. (2018). Von der reflexiven Methodologie zur empirischen Wissenschaftstheorie. In: L. Akremi et al. Hrsg. Handbuch Interpretativ Forschen. Weinheim, Basel: Beltz Juventa, 226-244.

Knoblauch, H. (im Druck). Reflexive Methodologie und empirische Wissenschaftstheorie. In: M. Pfadenhauer und E. Scheibelhofer. Hrsg. Interpretative Sozialforschung: die Entwicklung in Wien. Methodologie, Methoden und empirische Beiträge zur Organisationssoziologie. Wiesbaden: Springer.

Knorr Cetina, K. (1984). Die Fabrikation von Erkenntnis: Zur Anthropologie der Naturwissenschaft. Frankfurt/Main: Suhrkamp.

Kuhn, T. S. (1967). Die Struktur wissenschaftlicher Revolutionen. Frankfurt/Main: Suhrkamp. 
Latour, B. und S. Woolgar (1979). Laboratory Life: The Social Construction of Scientific Facts. Beverly Hills: Sage.

Merton, R. K. (1972). Wissenschaft und demokratische Sozialstruktur. In: P. Weingart. Hrsg. Wissenschaftssoziologie 1: Wissenschaftliche Entwicklung als sozialer Prozess. Frankfurt/Main: Fischer, 45-59.

Mittelstraß, J. (1974). Das normative Fundament der Sprache. In: idem. Hrsg. Die Möglichkeit von Wissenschaft. Frankfurt/Main: Suhrkamp, 158-205.

Mulkay, M. (1979). Science and the Sociology of Knowledge. London: G. Allen \& Unwin.

Münch, R. (2007). Die akademische Elite: Zur sozialen Konstruktion wissenschaftlicher Exzellenz. Frankfurt/Main: Suhrkamp.

Popper, K. (1935). Logik der Forschung: Zur Erkenntnistheorie der modernen Naturwissenschaft. Wien: Springer.

Schickore, J. und F. Steinle (2006). Introduction: Revisiting the Context Distinction. In: idem. Hrsg. Revisiting Discovery and Justification. Historical and Philosophical Perspectives on the Context Distinction. Dordrecht: Springer [DOI: 10.1007/1-4020-4251-5], vii-xix.

Schmitt, F. Hrsg. (1994). Socializing Epistemology: The Social Dimension of Knowledge. Boston, London: Rowman \& Littlefield.

Stehr, N. (2002). Wissenspolitik: Die Überwachung des Wissens. Frankfurt/Main: Suhrkamp.

Stengers, I. (2008). Der kosmopolitische Vorschlag. In: B. Latour und I. Stengers. Hrsg. Spekulativer Konstruktivismus. Berlin: Merve, 153-186.

Weingart, P. (2003). Wissenschaftssoziologie. Bielefeld: transcript.

Wilke, R. und Hill, M. (2019). On New Forms of Science Communication and Communication in Science: A Videographic Approach to Visuality in Science Slams and Academic Group Talk. Qualitative Inquiry [DOI: 10.1177/1077800418821531], 1-16.

Wilke, R., E. Lettkemann und H. Knoblauch (2016). Präsentationales Wissen. In: idem. Hrsg. Knowledge in Action: Neue Formen der Kommunikation in der Wissensgesellschaft. Wiesbaden: Springer [DOI: 10.1007/978-3-658-18337-0], 239-271. 
